\title{
Review of: "Simulation of gas sensing mechanism of porous metal oxide semiconductor sensor based on finite element analysis"
}

\author{
Tong Zhang ${ }^{1}$ \\ 1 Jilin University
}

Potential competing interests: The author(s) declared that no potential competing interests exist.

In this manuscript, the authors used computer software -- COMSOL Multiphysics Tool to simulate the gassensing process of acetone detection. Mesoporous $\alpha-\mathrm{Fe}_{2} \mathrm{O}_{3}$ is selected as the research object. Based on the gas absorption and desorption model, the author has modeled the test system, gas-sensitive reaction parameters, and gas-sensitive materials. The simulation results have been compared with the real measurement results to verify the correctness of the simulation. I recommend a major revision.

However, there are still several questions need to be explained clearly as follows:

1. Typically, as a biomarker of diabetes, acetone detection with metal oxide semiconductors (MOSs) has attracted much attention. As illustrated in the precious works, several MOSs are reported to be sensitive to acetone, such as $\ln _{2} \mathrm{O}_{3}, \mathrm{ZnO}$, and $\mathrm{SnO}_{2}$. Although $\alpha-\mathrm{Fe}_{2} \mathrm{O}_{3}$ is also one of the most promising gassensing materials, why do the authors choose $\alpha-\mathrm{Fe}_{2} \mathrm{O}_{3}$ as a sample to detect acetone?

2. As the authors mentioned, the purpose of simulation is to provide guidance for the design of gassensitive materials. However, according to the manuscript, the authors did not highlight which gassensing parameter is the key to the acetone sensing process.

3. As a class of defects in MOSs, oxygen vacancies also play a vital role in surface reaction. Therefore, it is reasonable to take oxygen vacancies into account in simulation. Moreover, $\alpha-\mathrm{Fe}_{2} \mathrm{O}_{3}$ is a kind of polyvalent metallic compounds and both $\mathrm{Fe}^{2+}$ and $\mathrm{Fe}^{3+}$ existing in the matrix. Thus in gas sensing processed, changes in metal valence states should also be considered.

4. More gases should be tested to measure the selectivity.

5. The definition of response, response and recovery time should be mentioned.

6. There are many abbreviations in the manuscript, such as COMSOL, SAW and BAR. Please give the full name corresponding to the abbreviation.

7. "Here we use formula (10) to calculate the fitting index between the experimental data and the simulated data." "formula (10)" should be "formula (11)". 